\title{
Family history is neglected in the work-up of patients with colorectal cancer: a quality assessment using cancer registry data
}

\author{
D. A. van Dijk · M. J. Oostindiër · W. M. Kloosterman-Boele • \\ P. Krijnen - H. F. A. Vasen - Hereditary Tumor Study Group of the \\ Comprehensive Cancer Centre West (CCCW)
}

Received: 5 December 2006/ Accepted: 6 December 2006/Published online: 20 January 2007

(C) Springer Science+Business Media B.V. 2007

\begin{abstract}
In the diagnostic work-up of hereditary non-polyposis colorectal cancer (HNPCC, Lynch syndrome), high-risk patients can be identified using information from the family history on cancer ('Amsterdam criteria' and 'Bethesda guidelines'). To investigate to what extent the medical specialists apply these criteria to patients with colorectal carcinoma and a suspicion of HNPCC, we collected information on diagnostic work-up of 224 patients of seven hospitals in the region of the Comprehensive Cancer Centre West in Leiden, The Netherlands. These patients were diagnosed with colorectal cancer between 1999 and 2001 and satisfied at least one of the Bethesda guidelines. A complete family history was recorded for 38 of the 244 patients $(16 \%)$. Patients with a complete family
\end{abstract}

Hereditary Tumour Study Group: Leiden University Medical Centre: Dr. H.J. Gelderblom, Medical Oncologist; Dr. H.H. Hartgrink, Oncological Surgeon; Dr. F.J. Hes, Clinical Geneticist; Dr. J. Morreau, Pathologist; Dr. H.F.A. Vasen, Gastro-enterologist. Diaconessenhuis Leiden: Dr. L.F.S.J. Crobach, Gastro-enterologist.

D. A. van Dijk

The Netherlands Foundation for the Detection of

Hereditary Tumours, Leiden University Medical Centre,

Leiden, The Netherlands

M. J. Oostindiër · W. M. Kloosterman-Boele .

P. Krijnen

Comprehensive Cancer Centre West, Leiden,

The Netherlands

H. F. A. Vasen ( $\square)$

Department of Gastroenterology, Leiden University

Medical Centre and The Netherlands Foundation for the

Detection of Hereditary Tumours, Poortgebouw Zuid,

Rijnsburgerweg 10, 2333 AA Leiden, The Netherlands

e-mail: hfavasen@stoet.nl history were more likely to be referred to the Clinical Genetic Centre than those with an incomplete or absent family history $(53 \%$ vs. $13 \%$ and $4 \%$, respectively; $P<0.0001$ ), and more likely to be analyzed for microsatellite instability (MSI), which is a characteristic of HNPCC (34\% vs. $6 \%$ and $1 \%$, respectively; $P<0.0001)$. We conclude that the family history is neglected in the majority of patients with colorectal cancer and MSI-analysis is only performed in a small proportion of the patients that meet the guidelines for this analysis.

Keywords Bethesda criteria - Colorectal cancer . Family history $\cdot$ Hereditary non-polyposis colorectal cancer - Lynch syndrome - Microsatellite instability

\section{Abbreviations \\ $\mathrm{CCCW}$ Comprehensive cancer centre west \\ CR Cancer registry \\ HNPCC Hereditary non-polyposis colorectal cancer MSI Microsatellite instability}

\section{Introduction}

Colorectal cancer (CRC) is one of the most common forms of cancer in Western society. Every year 9,500 patients in the Netherlands are diagnosed with the disease and almost half of them die from it (Dutch Cancer Registry, 2002). It is estimated that in around $20 \%$ of the patients with a colorectal tumour genetic factors play a role in the aetiology [1]. About $1-5 \%$ of the patients with CRC are thought to have hereditary non-polyposis colorectal cancer (HNPCC, Lynch 
Syndrome); a dominant hereditary disease, which is caused by a defect in one of the DNA-Mismatch Repair (MMR) genes [2]. The most important clinical characteristics of CRC associated with HNPCC, are the relatively young age at which patients are diagnosed with the disease (average $<45$ years old) and the proximal localisation of the tumour in the colon. Besides an increased risk of developing a tumour in the colon, there is an increased risk of developing a tumour elsewhere in the body, especially in the endometrium (lifetime risk: $50 \%$ ), the small intestines, the ovaries, the brain, the urinary tract, the biliary tract and the development of a keratoacanthoma or a carcinoma of the sebaceous glands of the skin [3].

The identification of patients with hereditary colorectal carcinoma is of great importance for the patient, because the treatment and follow-up of the tumour differ from those with non-hereditary colorectal carcinoma [4]. Furthermore, the identification of these patients is important, because it offers efficient manners for the prevention of colorectal carcinoma and other forms of cancer for the patient himself as well as his family [5]. It has been shown that a colonoscopy every three years can lead to a decline in mortality of at least $65 \%$ [6].

The genetic defect in HNPCC can be detected by immunohistochemic staining of the MMR proteins in tumour cells [7]. Another method to select families for mutation analysis of the MMR genes is analysis of errors in repetitive DNA-sequences, i.e., Micro Satellite Instability (MSI) [8]. MSI is found in around $15 \%$ of the non-selected CRC and in more than $95 \%$ of the colorectal tumours associated with HNPCC [9]. In 1996, guidelines were formulated for patients whose tumours should be analyzed for MSI [10]. These so-called Bethesda-guidelines describe practically all situations, where there is a suspicion of HNPCC. Recently these guidelines have been revised (Table 1) [11].

Before the discovery of the MMR-genes, the most common approach in the diagnostic work-up for HNPCC was to use the 'Amsterdam criteria'. These criteria are met if there are, within one family, three individuals with a colorectal (or another HNPCCassociated kind of) tumour, of whom one person is a first degree family member of the other two and at least one carcinoma is diagnosed before the age of fifty [12]. To evaluate the Amsterdam criteria in patients with CRC, a complete history on cancer in the patient's family has to be obtained. Until now it is not known whether an adequate family history is taken of all patients with a colorectal carcinoma. We also do not know to what extent medical specialists use the above mentioned clinical Bethesda guidelines and if the
Table 1 Guidelines for the performance of MSI-analysis of colorectal tumour [11]

Revised Bethesda-guidelines

A person with colorectal carcinoma diagnosed at age $\leq 50$

A person with colorectal carcinoma and MSI-associated pathology ${ }^{\mathrm{a}}<60$ years

A person with colorectal carcinoma and a HNPCC associated tumour ${ }^{\mathrm{b}}$

A person with colorectal carcinoma and a first degree relative with a colorectal or HNPCC associated tumour; at least one of the tumours is diagnosed before the age of 50

Three relatives diagnosed with colorectal carcinoma or a HNPCC associated tumour, diagnosed at any age; one patient needs to be a first degree relative of the other two

a The presence of tumor-infiltrating lymphocytes, so called, "Crohn's like lymfocyte reaction", mucinous or signet ring cell carcinoma differentiation or medullary growth pattern

b Carcinoma of the endometrial tissue, stomach, small intestines, pancreatic gland, biliary tract, urinary tract, ovaries, brain, keratoacanthoma and carcinoma of the sebaceous glands

tumours of all the patients who match the criteria are tested for MSI. The objective of the present study was to answer these questions using data of the Cancer Registry of the Comprehensive Cancer Centre West (CCCW) in Leiden, The Netherlands.

\section{Patients and methods}

We selected patients who were diagnosed with a primary and invasive colorectal tumour in the period 1999-2001 from the Cancer Registry (CR) of the Comprehensive Cancer Centre West (CCCW) in The Netherlands. The patients had to satisfy one of the following two Bethesda guidelines: the patient had to have more than one tumour, i.e., one colorectal carcinoma and a second one (colorectal cancer or another HNPCC-associated kind of tumour), or the patient had to be fifty years or younger at diagnosis. The selected patients were considered to have an indication for the performance of MSI-analysis and/or referral to the Clinical Genetic Centre (CGC). Patients with a carcinoma in situ or a carcinoïd of the appendix were not included in the analysis.

Between 1999 and 2001, 434 patients who complied with the above mentioned criteria were diagnosed with $\mathrm{CRC}$ in one of the twelve hospitals in the CCCWregion. Seven hospitals gave permission for the collection of information concerning family history, MSI-analysis and referral to the CGC. We extracted this information from the various (electronic) medical reports. The family history was considered complete if the medical records reported on cancer in the family, and if so, information about the age at the time of the 
Table 2 Diagnostic work-up for HNPCC in 244 patients with colorectal cancer, by completeness of the family history as reported in the medical records

\begin{tabular}{llll}
\hline Diagnostic workup & $\begin{array}{l}\text { Family history } \\
\text { complete }(n=38)\end{array}$ & $\begin{array}{l}\text { Family history } \\
\text { incomplete }(n=136)\end{array}$ & $\begin{array}{l}\text { Family history } \\
\text { absent }(n=70)\end{array}$ \\
\hline Referred to CGC & $20(53 \%)$ & $17(13 \%)$ & $3(4 \%)$ \\
MSI-analysis performed & $13(34 \%)$ & $8(6 \%)$ & $1(1 \%)$ \\
Results of MSI-analysis & 3 MSI, 10 stable & 7 stable, 1 unknown & 1 stable \\
Diagnosis of HNPCC & $6(16 \%)$ & $3(2 \%)$ & $1(1 \%)$ \\
\hline
\end{tabular}

diagnosis, the type of cancer and the occurrence of cancer within first-degree and second-degree family members.

Data were collected of 244 patients. Of these patients, 120 patients had multiple tumours, 109 patients were fifty years or younger at the time of diagnosis, and 15 patients had both characteristics. For comparisons between patients with multiple tumours and patients who were young at diagnosis, those with both characteristics were allocated to the "multiple tumours" group. The data were analyzed using SPSS statistical software (version 12.0.1). Univariate comparisons of proportions between patient groups were performed by Chi-squared test. Multivariate logistic regression analysis was used to study whether the presence of a complete family history or referral to a CGC could be explained by age, sex, inclusion criterion (multiple tumours or young age at diagnosis), hospital or type of medical specialist.

\section{Results}

The study group consisted of 244 persons, who complied with one of the Bethesda guidelines and therefore were considered to be referred for MSI-analysis and/or genetic counselling. The male:female ratio was 49:51 and did not differ between the groups selected on the basis of multiple tumours or age $\leq 50$ years at diagnosis.

A complete family history was recorded in the medical records of $38(16 \%)$ of the 244 patients. For 136 patients $(55 \%)$ limited information on the family history was available, and for 70 (29\%) patients no information on the family history was found in the medical records. In the seven participating hospitals, a family history was reported for $38-91 \%$ of the patients.

Of the 38 patients with a complete family history, 20 $(53 \%)$ were referred to the CGC. This percentage was higher than that of patients with an incomplete family history $(13 \%)$ and that of patients without any information on family history $(4 \%)(P<0.0001$, Table 2$)$. MSI-analysis was performed more often in the patients with a complete family history: $34 \%$ of patients with a complete family history compared to $6 \%$ of patients with an incomplete family history and $1 \%$ of patients without any family history $(P<0.0001)$ (Table 2$)$. Presence of a complete family history and the performance of MSI-analysis were not associated with age, sex, inclusion criterion (multiple tumours or young age at diagnosis), hospital or type of medical specialist (multivariate logistic regression analysis; data not shown).

\section{Discussion}

We used the Bethesda-guidelines to select a group of patients with a suspicion of HNPCC. These patients were diagnosed with colorectal cancer between 1999 and 2001, a period during which MSI-analysis and the Bethesda guidelines were already available. Therefore we expected that for these patients, physicians would have examined and reported their patients' family history and that MSI-analysis would have been performed. In our study group, however, the family history of the patients diagnosed with colorectal carcinoma was not sufficiently examined and reported in the medical records. For this reason, we believe the Bethesda-guidelines were not sufficiently applied by the physicians. As a consequence, MSI-analysis was performed on a small proportion of the tumours. More patients with a complete family history in their medical records were referred by their physicians to the CGC than patients without such a family history. MSI-analysis was also performed more often in this group. We expect that in a low-risk population, i.e., patients with colorectal cancer who do not meet the Bethesda guidelines, these results would be even more dramatic.

On the one hand, our results may appear to be better than they actually are. We collected our data using medical records from various medical specialties, while the treating physician will not have this overview in practice. On the other hand, it is possible that when a physician examined a family history and none of the family members was diagnosed with cancer, he did not report it in the medical records. In this case, the family history was considered as absent, although it in fact was examined. Nevertheless we expect that if MSI-analysis was performed or the patient was referred to the CGC, this would have certainly been reported. 
We found that the attention for HNPCC in the diagnostic workup for CRC differed widely. For the seven participating hospitals, the proportion of patients with a reported family history on cancer ranged from $38 \%$ to $91 \%$. Furthermore, only half of the approached hospitals were willing to cooperate. For these reasons, we cannot generalise our results for the whole CCCWregion. Nevertheless, we conclude that the family history appears to be neglected in the majority of patients with colorectal cancer in our study period, and that MSI-analysis was only performed in a small proportion of the patients that meet the guidelines for this analysis. Possibly, the attention for identification of patients with HNPCC has increased in more recent years. Our findings underscore the importance of implementation of family history and Bethesda guidelines in the physician education.

Acknowledgements The authors thank the physicians from the seven participating hospitals in the CCRW-region for permission to collect the data of their patients from the medical records.

\section{References}

1. Lynch HT, de la Chapelle A (2003) Hereditary colorectal cancer. N Engl J Med 348:919-932

2. Peltomaki P, Vasen H (2004) Mutations associated with HNPCC predisposition-Update of ICG-HNPCC/INSiGHT mutation database. Dis Markers 20:269-276

3. Vasen HF (2000) Clinical diagnosis and management of hereditary colorectal cancer syndromes. J Clin Oncol 18(suppl):81S-92S
4. de Vos tot Nederveen Cappel WH, Buskens E, van Duijvendijk $\mathrm{P}$ et al (2003) Decision analysis in the surgical treatment of colorectal cancer due to a mismatch repair gene defect. Gut 52:1752-1755

5. de Vos tot Nederveen Cappel WH, Nagengast FM, Griffioen $\mathrm{G}$ et al (2002) Surveillance for hereditary nonpolyposis colorectal cancer: a long-term study on 114 families. Dis Colon Rectum 45:1588-1594

6. Jarvinen HJ, Aarnio M, Mustonen $\mathrm{H}$ et al (2000) Controlled 15 year trial on screening for colorectal cancer in families with Hereditary Non-Polyposis Colorectal Carcinoma. Gastroenterology 118:969-971

7. De Jong AE, van Puijenbroek M, Hendriks Y et al (2004) Microsatellite instability, immunohistochemistry, and additional PMS2 staining in suspected hereditary nonpolyposis colorectal cancer. Clin Canc Res 10:972-980

8. Kievit W, de Bruin JH, Adang EM et al (2005) Cost effectiveness of a new strategy to identify HNPCC patients. Gut 54:97-102

9. Menko FH, Griffioen G, Wijnen JT et al (1999) Genetics of colorectal cancer. I. Non-polyposis and polyposis forms of hereditary colorectal cancer. Ned Tijdschr Geneeskd 143:1201-1206

10. Rodriguez-Bigas MA, Boland CR, Hamilton SR et al (1997) A National Cancer Institute Workshop on Hereditary Nonpolyposis Colorectal Cancer Syndrome: meeting highlights and Bethesda guidelines. J Natl Canc Inst 89:1758-1762

11. Umar A, Boland CR, Terdiman JP et al (2004) Revised Bethesda Guidelines for hereditary nonpolyposis colorectal cancer (Lynch syndrome) and microsatellite instability. J Natl Cancer Inst 96:261-268

12. Vasen HF, Watson P, Mecklin JP, Lynch HT (1999) New clinical criteria for hereditary nonpolyposis colorectal cancer (HNPCC, Lynch syndrome) proposed by the International Collaborative group on HNPCC. Gastroenterology 116:1453-1456 